\title{
Higher-Order Numerical Solutions of the Quarter Car Suspension Model
}

\author{
Albert Materdey \\ Quantum FC, Inc. \\ Boston, MA, USA \\ materdey@ieee.org
}

\begin{abstract}
Car suspension was studied using the Quarter Car Suspension Model, which consists of a suspension spring, a damper, and a tire spring that holds a quarter of a car. Applying Newton's second law to the sprung and un-sprung masses, the Quarter Car Suspension mathematical model was derived as a system of two coupled second-order differential equations. This system was rearranged into a system of four coupled first-order differential equations. Algorithms were derived to solve this system in the time domain using the Euler's, second-order Runge-Kutta , and fourth-order Runge-Kutta methods. Matlab was used to obtain numerical solutions and computer animations by varying the suspension and tire spring constants, and the damping constants for different road changes: steps, bumps, and holes. We found that decreasing the spring constants and increasing the damping constant improved the ride, and overall bumps are a little bit easier to handle than steps and holes. Larger bumps or holes are more difficult to traverse, as well as having certain bump-bump or bump-hole separation. For larger values of the suspension and tire spring constants, for example in heavier vehicles with large tires such as utility, crane, and backhoe trucks, higher order algorithms are essential to describe correctly the high-frequency oscillations of the vehicle suspension system.
\end{abstract}

Keywords: Quarter Car Suspension, Coupled Differential Equations, Numerical Solutions, Second And Fourth Order Runge-Kutta, Numerical Algorithms, Computer Animations, Tire Spring, Damping; Road Holes, Road Bumps, Road Steps, Trucks.

\section{Introduction}

Our goal is to study a car suspension as a system of damped harmonic oscillators using Matlab. We developed an algorithm that solves the system of damped harmonic oscillators using the Euler's method or the Runge-Kutta's second (RK2) and fourth-order (RK4) methods. By varying the spring and damping constants, which describe the springs and shock absorber, we hope to determine what suspension is best for a given car and what is the maximum speed you can go over a bump so your car will not bottom out.

The Quarter Car Suspension Model describes the mass of the axle/suspension and the wheel as a bob and spring system, respectively, which is coupled to the upper quarter car and suspension which are described with the upper bob, spring and damper, also respectively. The math description is a system of two coupled second-order ordinary differential equations (ODEs).

A first-order differential equation can be solved numerically using algorithms that are written in algebraic equations such as the Euler's algorithm or the Runge-Kutta's second (RK2) and fourth-order (RK4) algorithms. For example, an Euler's algorithm can be written to solve a single or a system of two first-order differential equations. By writing the second-order differential equation for a damped harmonic oscillator as a system of two coupled first-order ones, it can be solved using the Euler's algorithm for a system of two first-order ODEs.

Each second-order ODE was solved using two coupled first-order ODEs. The numerical solution of the Quarter Car Suspension Model consisted of an algorithm that solves the four coupled first-order ODEs. This system was solved using Euler and the RK2 and RK4 algorithms.

\section{Car Suspension}

There are two main types of car suspension; dependent and independent. A dependent suspension has a live axle ( 2 for four wheel drive), as well as a dead axle for trucks and vans. The axles can also be rigid to provide more stability. An 
independent suspension is independently attached to the body or sub-frame. Different spring combinations are possible. When driven wheels are independently suspended, the differential is fixed to the frame and drives the wheels by joined drive shafts. In this paper we study one independent suspension that holds a quarter of the vehicle's weight.

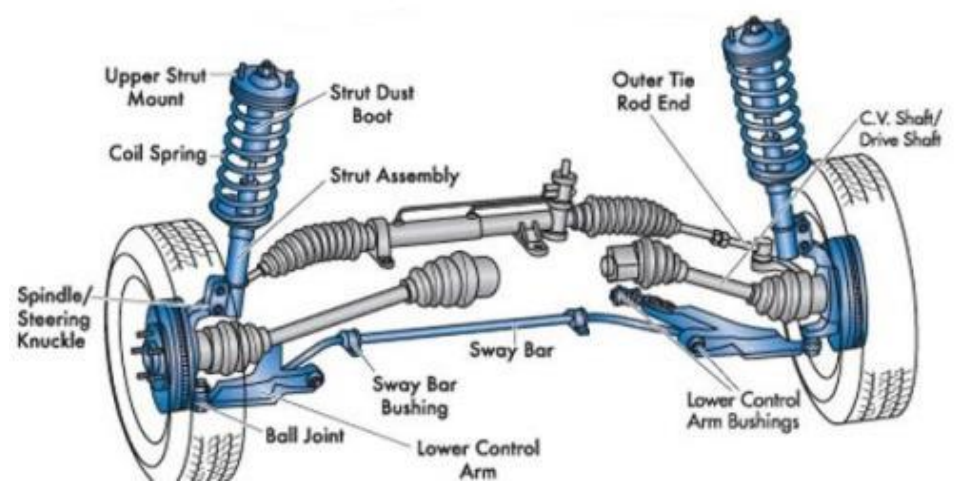

Fig. 1: This figure depicts a real example of car suspension [8], which was simulated using Matlab.

\section{Quarter Car Suspension Model}

\subsection{Diagram}

The Quarter Car Suspension Model, see Figure 2, describes the suspension as a spring, of spring constant $K_{s}$, and a damper, or shock absorber, of damping constant $b$, mounted between the quarter weight of the car, of mass $m_{2}$, and the unsprung mass, which consists of the axle, suspension, and wheel, of mass $m_{2}$. The tire acts as a second spring, of spring constant $K_{1}$, mounted between the unsprung mass $m_{1}$ and the road. The vertical position of $m_{1}$ is $\mathrm{x}$, the vertical position of $m_{2}$ is $\mathrm{y}$, while the vertical position of the road is $\mathrm{u}$. Variations in the road surface will affect the suspension system changing the vertical positions and velocities of the masses $m_{1}$ and $m_{2}$, which are $x(t), y(t), \dot{x}(t), \dot{y}(t)$, respectively. At any given time, the length of the suspension spring is $(y-x)$, and that of the tire spring is $(x-u)$. The length of a spring determines its recovery force as described by Hooke's Law.

When the suspension goes over a hole in the road, the system made up of $K_{s}$ and $b$ is stretched. $m_{2}$ is pulled down on (negative forces by $K_{S}$ and $b$ ) while $m_{l}$ is being pulled up (positive forces by $K_{s}$ and $b$ ), $K_{w}$ is stretched to pull $m_{l}$ downwards (negative force by $K_{w}$ ). The free body diagrams of forces acting on $m_{1}$ and $m_{2}$ are shown in the next section.

\section{Quarter Car Suspension}

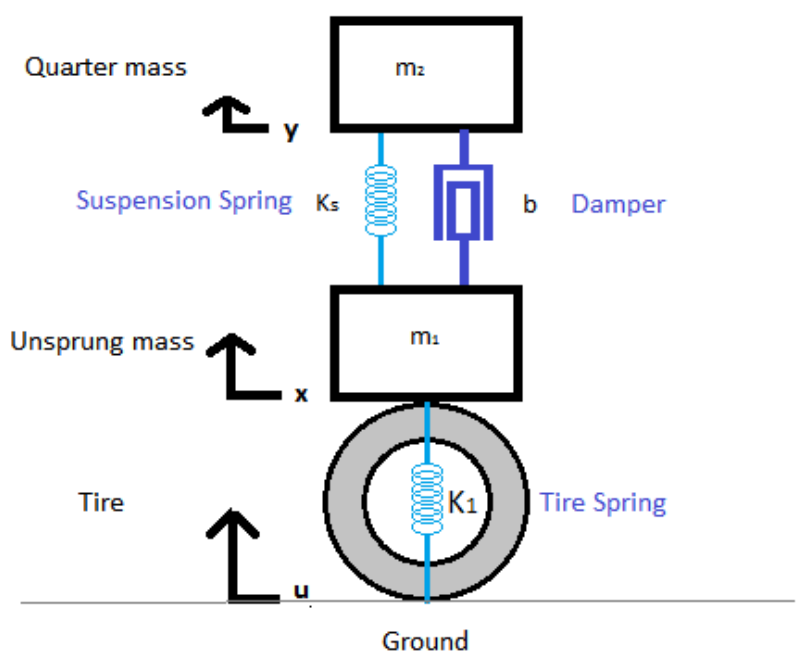

Fig. 2: The Quarter Car Suspension Model. 


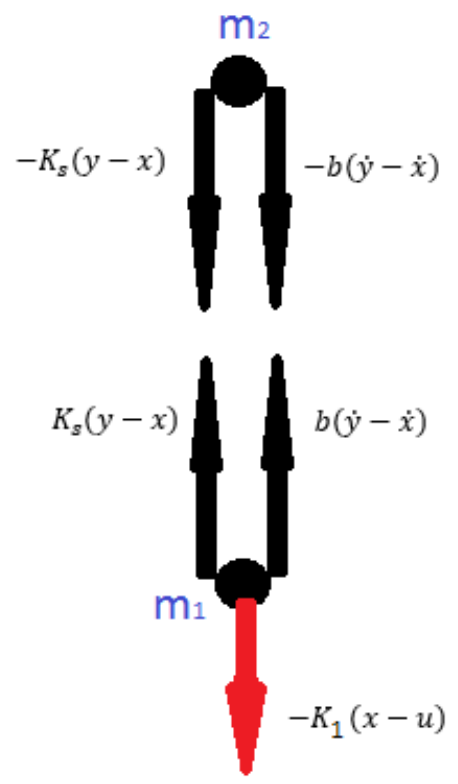

Fig. 3: This is a diagram of the forces acting on $m_{1}$ and $m_{2}$ by the car suspension system.

\subsection{Free Body Diagram}

When the suspension system is stretched, the net force on $m_{2}$ consists of a superposition of two forces pointing in the downward direction: $-K_{s}(y-x)$ and $-b(\dot{y}-\dot{x})$. The net force on $m_{1}$ consists of a superposition of three forces, two pointing in the upward direction: $K_{s}(y-x)$ and $b(\dot{y}-\dot{x})$, and the third one pointing in the downward direction: $-K_{1}(x-$ $u$ ). The free-body diagram for each mass is shown in Figure 3.

\subsection{Newton's Second Law}

According to Newton's Second Law, the net force acting on an object of mass $m$ equals $m a$, where $a$ is the acceleration of the object.

For $m_{2}$ Newton's Second law reads

$$
F_{\text {net }}=m a
$$

$$
\begin{gathered}
-K_{s}(y-x)-b(\dot{y}-\dot{x})=m_{2} \ddot{y} \\
m_{2} \ddot{y}+K_{s} y+b \dot{y}=K_{s} x+b \dot{x}
\end{gathered}
$$

For $m_{1}$ Newton's Second law reads

$$
\begin{aligned}
& K_{s}(y-x)+b(\dot{y}-\dot{x})-K_{1}(x-u)=m_{1} \ddot{x} \\
& m_{1} x+b \dot{x}+\left(K_{s}+K_{1}\right) x=K_{s} y+b \dot{y}+K_{1} y
\end{aligned}
$$

So the quarter car suspension is described by two coupled second order ODEs, equations (3), and (5).

\section{Rewriting Each Second Order ODE as a System of Two Coupled First-Order ODEs}

The system of two coupled second order ODEs derived in the previous section is now rewritten as a system of four coupled first order ODEs. 
1.

$$
\begin{gathered}
\dot{x}=x_{1} \\
\ddot{x}=\dot{x}_{1}=\frac{K_{s}}{m_{1}} y+\frac{b}{m_{1}} y+\frac{K_{1}}{m_{1}} u-\frac{b}{m_{1}} x-\frac{K_{s}+K_{1}}{m_{1}} x \\
\dot{x}_{1}=\frac{K_{s}}{m_{1}} y+\frac{b}{m_{1}} y_{1}+\frac{K_{1}}{m_{1}} u-\frac{b}{m_{1}} x_{1}-\frac{K_{s}+K_{1}}{m_{1}} x
\end{gathered}
$$

3.

$$
\begin{gathered}
\dot{y}=y_{1} \\
\ddot{y}=\dot{y}_{1}=\frac{K_{s}}{m_{2}} x+\frac{b}{m_{2}} x+\frac{K_{s}}{m_{2}} y-\frac{b}{m_{2}} y \\
\dot{y}_{1}=\frac{K_{s}}{m_{2}} x+\frac{b}{m_{2}} x_{1}+\frac{K_{s}}{m_{2}} y-\frac{b}{m_{2}} y_{1}
\end{gathered}
$$

\section{Numerical Solutions}

\subsection{Taylor Series Expansion}

$$
\begin{aligned}
& y(x+\Delta x, t+\Delta t)= y(x, t)+\frac{\partial y}{\partial x} \Delta x+\frac{\partial y}{\partial t} \Delta t \\
&+\frac{1}{2} \frac{\partial^{2} y}{\partial x^{2}} \Delta x^{2}+\frac{1}{2} \frac{\partial^{2} y}{\partial t^{2}} \Delta t^{2}+\frac{\partial^{2} y}{\partial x \partial t} \Delta x \Delta t \\
&+\frac{1}{6} \frac{\partial^{3} y}{\partial t^{3}} \Delta x^{3}+\frac{1}{6} \frac{\partial^{3} y}{\partial x^{2} \partial t} \Delta t^{3} \\
&+\frac{1}{2} \frac{\partial^{3} y}{\partial x^{2} \partial t} \Delta x^{2} \Delta t+\frac{1}{2} \frac{\partial^{3} y}{\partial x \partial t^{2}} \Delta x \Delta t^{2}+\cdots \\
& \mathrm{y}(x+\Delta x, t+\Delta t)=\sum_{n=0}^{\infty} \frac{\partial^{n+k} y}{\partial x^{n+k}} \sum_{k=0}^{\infty} \frac{\partial^{n+k} y}{\partial x^{n+k}} \frac{\Delta x^{n}}{n !} \frac{\Delta t^{k}}{k !}
\end{aligned}
$$

\subsection{Euler's Method}

Euler's algorithm results from solving the first-order ODE

$$
\frac{d x}{d t}=f(x, t)
$$

using a Taylor's series expansion including terms up to first-order approximation.

$$
x(t+\Delta t)=x(t)+\frac{\mathrm{dx}}{d t} \Delta t=x(t)+f \cdot \Delta t
$$

\subsection{Higher Order Methods: Runge-Kutta 2 and 4}

Runge-Kutta 2 or RK2 algorithm results from solving the same first-order ODE, equation (12) using a Taylor's series expansion including terms up to second-order approximation.

$$
a_{1}=f \cdot \Delta t
$$




$$
\begin{aligned}
a_{2}=f\left(x(t)+f \cdot \frac{\Delta t}{2}, t+\frac{\Delta t}{2}\right) \cdot \Delta t & =f\left(x(t)+\frac{a_{1}}{2}, t+\frac{\Delta t}{2}\right) \cdot \Delta t \\
x(t+\Delta t) & =x(t)+a_{2}
\end{aligned}
$$

Runge-Kutta 4 or RK4 algorithm results from solving the same first-order ODE, equation (12) using a Taylor's series expansion including terms up to fourth-order approximation.

$$
\begin{gathered}
a_{1}=f \cdot \Delta t \\
a_{2}=f\left(x(t) \cdot \frac{a_{1}}{2}, t+\frac{\Delta t}{2}\right) \cdot \Delta t \\
a_{3}=f\left(x(t) \cdot \frac{a_{2}}{2}, t+\frac{\Delta t}{2}\right) \cdot \Delta t \\
a_{4}=f\left(x(t)+a_{3}, t+\Delta t\right) \cdot \Delta t \\
x(t+\Delta t)=x(t)+\frac{1}{6}\left(a_{1}+2 a_{2}+2 a_{3}+a_{4}\right)
\end{gathered}
$$

These higher-order algorithms provide higher-accuracy numerical solutions without requiring more memory when using smaller space and time increments.

\section{Numerical Results and Analysis}

Damping is defined as how oscillations of a system decay after a disturbance from static equilibrium. If the damping constant is sufficiently high compared to the spring constant, the mass could slowly return to its rest position without ever overshooting. This case is called over-damped. Commonly, the mass tends to go past its starting position, and then return, overshooting again. With each overshoot, some energy in the system is dissipated, and the oscillations die towards zero. This case is called under-damped. Between the over-damped and under-damped cases, there exists a certain level of damping at which the system will just fail to overshoot and will not make a single oscillation. This case is called critical damping. The key difference between critical damping and over damping is that, in critical damping, the system returns to equilibrium in the minimum amount of time.

Un-sprung weight $m_{1}$ is generally defined as the weight of the vehicle's components that are not supported by the springs. This includes tires, wheels, brakes, half the control arm's weight and other components. These components are then (for calculation purposes) assumed to be connected to a vehicle with no sprung mass.

Sprung weight $m_{2}$ is generall defined as the weight of the vehicle resting on the springs, not the total vehicle weight. Calculating this requires knowing the vehicle's sprung weight (total weight less the un-sprung mass), the front and rear roll center heights and the sprung center of gravity height (used to calculate the roll moment arm length).

$K_{S}$ is the suspension spring constant, $b$ is the damping constant, and $K_{1}$ is the tire spring constant.

I studied the suspension response for different road changes: step, bump, and hole, bump-bump, bump-hole, with different heights and separations. shown in Figures 6, 8, 10,11, and 12 respectively. Steps and bumps are defined with a positive step amplitude, while holes are defined with a negative step amplitude.

Analysis: An increase in the mass $m_{2}$ causes a larger increase in position and velocity compared to the changes in the unsprung mass $m_{1}$. Through the Matlab program we can assume optimal results for a car with standard wheels and suspension. This evidence is supported by the numerical results shown below, which I tested in the experimental phase of the project. The sprung mass $m_{2}$, when adjusted, has a more comfortable ride than when $m_{1}$, the unsprung mass is adjusted. The most comfortable results come from adjusting the damping constant $b$. 
Numerical values used to vary $K_{s}, b$, and $K_{1}$ for the first three road changes are listed in Table 1 . Values for $m_{2}$, and $m_{1}$ were 500, and 10, respectively. Their initial positions are 3, and 2.9, respectively.The initial velocities are assumed 0 . The simulation time runs between 0 and 25. Numerical solutions for these values for step, bump, and hole in the road are plotted in Figures 5, 7, and 9, respectively. For all three road changes, increasing the suspension spring constant $K_{S}$ or the tire spring constant $K_{1}$ causes an increase in the oscillation frequency of the unsprung mass $m_{1}$ and a rise in its position. This would cause an unsafe ride as $m_{1}$ may collide with $m_{2}$, resulting in a bottom-out effect. A decrease in the tire spring constant $K_{1}$, which can be achieved with a lower tire pressure, will improve the ride in detriment of the gas mileage. Among the three road changes, it is slightly easier to handle a bump vs. a hole or a step. Increasing the damping constant $b$ decreases the number of oscillations of the unsprung mass and lowers its position, preventing any collision between $m_{1}$ and $m_{2}$, improving the ride.

Going more into depth we found the numerical solutions for Figures 11-12 for the bump, bump-bump, and bump-hole road changes. For these the default values of $K_{s}, b, K_{1}, m_{2}$, and $m_{1}$ stayed the same, while the separation between bumpbump, and bump-hole, and the height of the bump or hole were manipulated. The results of these road changes, shown for each mass and algorithms (top=positions, bottom= velocities), indicate that it is more difficult to go over bumps or holes with a larger amplitude, or having separation closest to the length between the car suspension front to back.

To more clearly show the difference in accuracy of the Euler, RK2, and RK4 algorithms, the time step was increased from 0.1 to 0.2 , reducing the number of steps by half. The accuracy of Euler's method for this time step is reduced compared to those of the higher order algorithms, with the results farther away from each other around the local extremes of the velocity curves.

Table 1: Values for $\boldsymbol{K}_{s}$, b, and $\boldsymbol{K}_{1}$ used in the numerical solution of the quarter car suspension model. $\boldsymbol{m}_{1}=10$ and $\boldsymbol{m}_{2}=500$. Initial positions for $\boldsymbol{m}_{2}$ and $\boldsymbol{m}_{1}$ are 3 and 2.9, respectively. Initial velocities are 0 . Final time is 25.

\begin{tabular}{|c|c|c|c|c|}
\hline Numeric Values & \multicolumn{4}{|c|}{ Step, bump, and hole } \\
\hline Ks & 1 & 2 & 10 & 10 \\
\hline b & 0.5 & 2.5 & 5 & 0.5 \\
\hline K1 & 0.2 & 0.4 & 0.8 & 0.8 \\
\hline
\end{tabular}
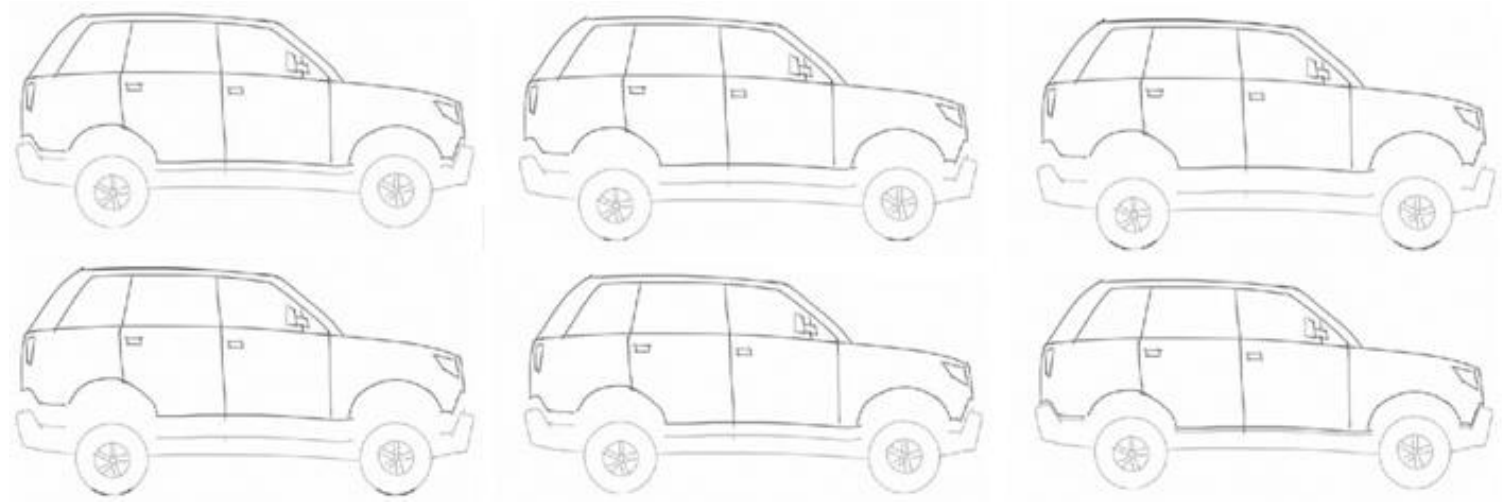

Fig. 4: Short movie clips were made that show the motion of the car and its axle/suspension as it hits different types of obstacles in the road. 


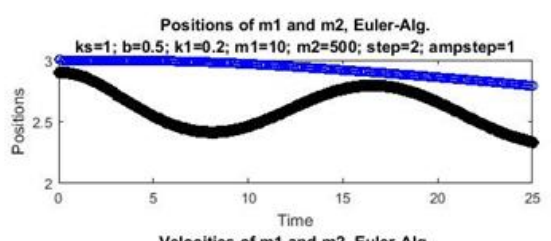

Velocities of $\mathrm{m} 1$ and $\mathrm{m} 2$. Euler-Alg



Positions of $\mathrm{m} 1$ and $\mathrm{m} 2$, Euler-Alg.

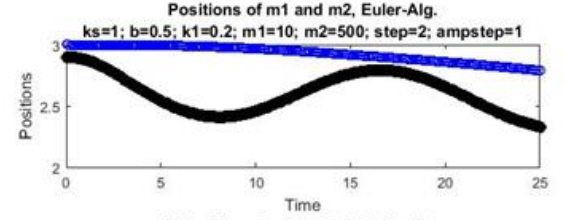

Velocities of $m 1$ and $m 2$, Euler-Alg.

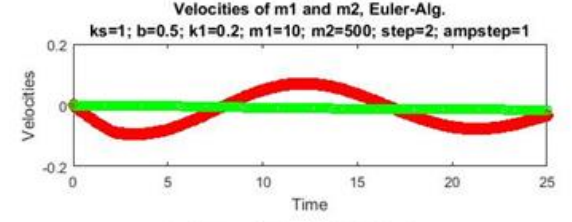

Positions of $\mathrm{m} 1$ and $\mathrm{m} 2$, Euler-Alg.
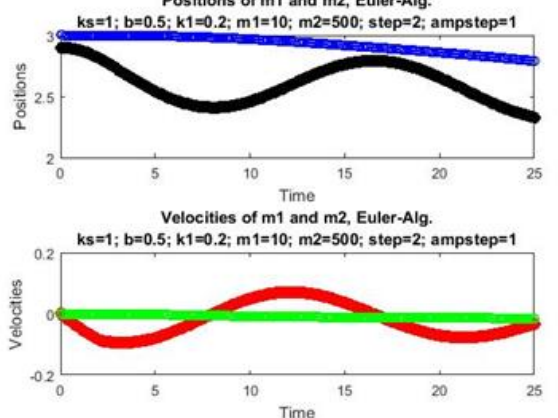

Positions of $m 1$ and $m 2$, Euler-Alg.
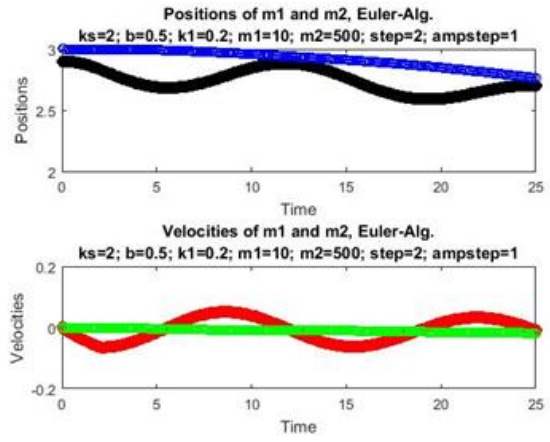

Positions of $\mathrm{m} 1$ and $\mathrm{m} 2$, Euler-Alg.

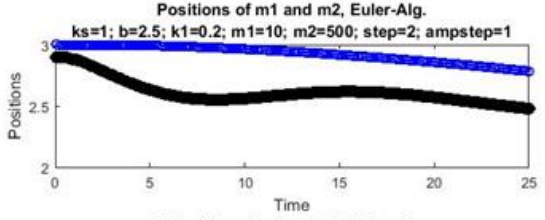

Velocities of $\mathrm{m} 1$ and $\mathrm{m} 2$, Euler-Alg.

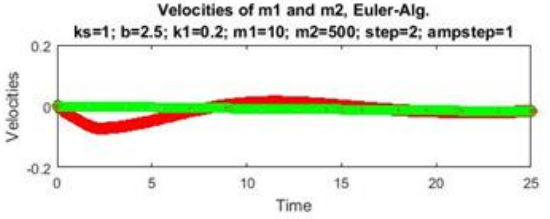

Positions of $\mathrm{m} 1$ and $\mathrm{m} 2$, Euler-Alg.
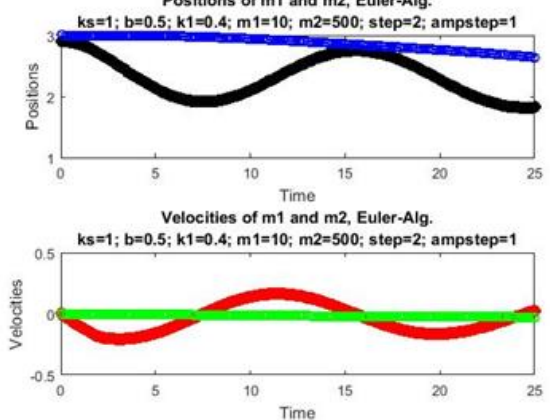

Positions of $\mathrm{m} 1$ and $\mathrm{m} 2$, Euler-Alg.
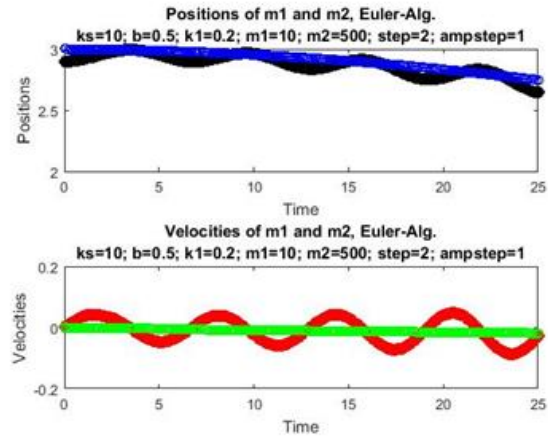

Positions of $\mathrm{m} 1$ and $\mathrm{m} 2$, Euler-Alg

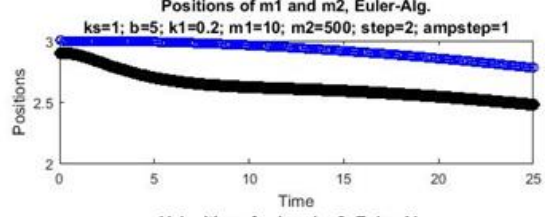

Velocities of $\mathrm{m} 1$ and $\mathrm{m} 2$, Euler-Alg

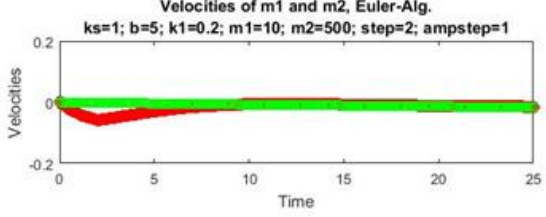

Positions of $\mathrm{m} 1$ and $\mathrm{m} 2$, Euler-Alg.

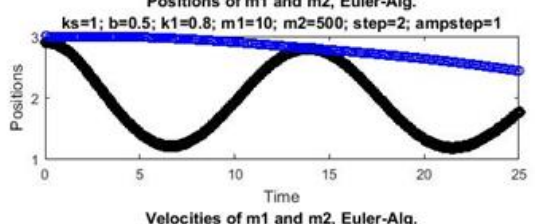

Velocities of $\mathrm{m} 1$ and $\mathrm{m} 2$, Euler-Alg.

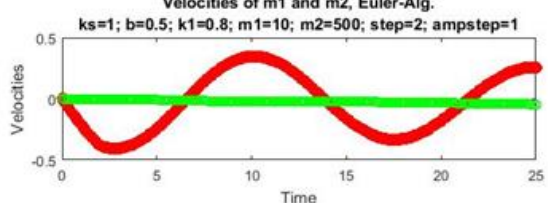

Fig. 5: Positions and velocities of $m_{1}$ and $m_{2}$ when $K_{s}$, b, and $K_{1}$ are varied respectively, when the vehicle hits a step in the road whose profile is shown in the next figure.



Fig. 6: Profile of a step in the road used in the numerical solution of the car suspension model whose results are shown in the previous figure. 


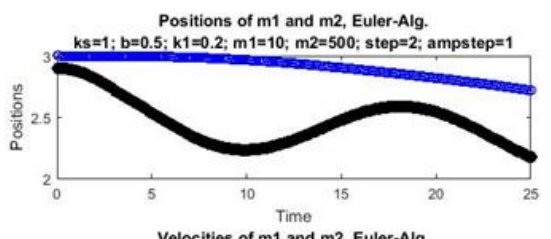

Velocitios of $m 1$ and $m 2$ Euler-Alg.

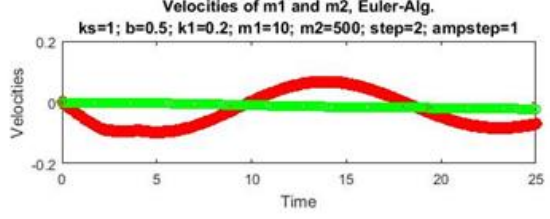

Positions of $\mathrm{m} 1$ and $\mathrm{m} 2$, Euler-Alg.

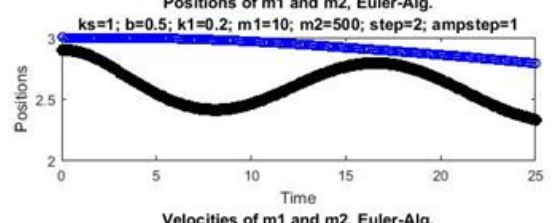

Velocities of $\mathrm{m} 1$ and $\mathrm{m} 2$, Euler-Alg.

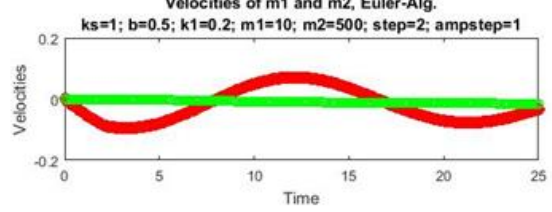

Positions of $m 1$ and $m 2$, Euler-Alg.
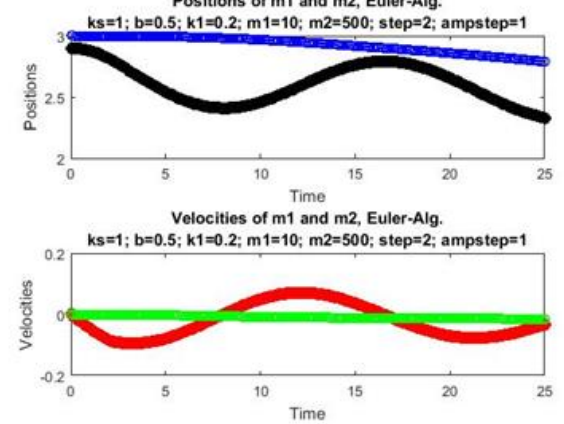

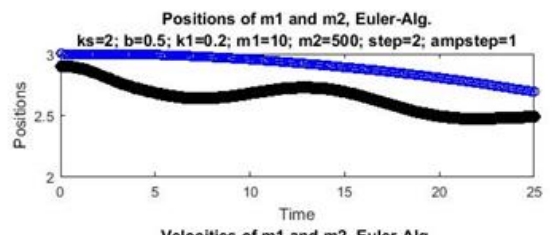

Velocities of $\mathrm{m} 1$ and $\mathrm{m} 2$, Euler-Alg.

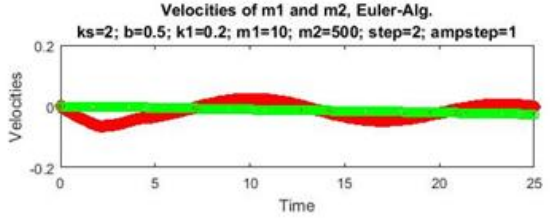

Positions of $\mathrm{m} 1$ and $\mathrm{m} 2$, Euler-Alg.


Positions of $\mathrm{m} 1$ and $\mathrm{m} 2$, Euler-Alg.
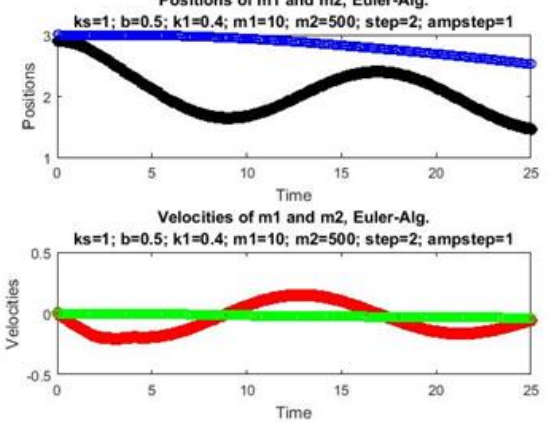
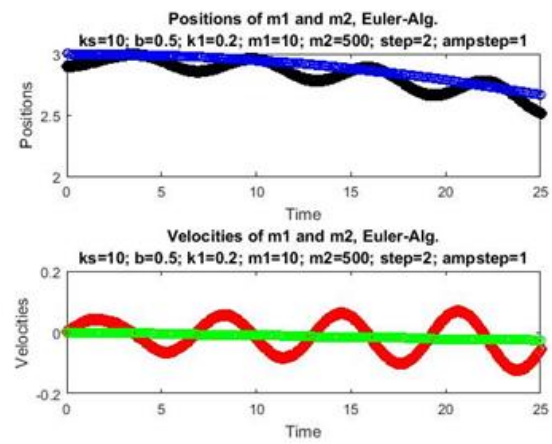

Positions of $\mathrm{m} 1$ and $\mathrm{m} 2$, Euler-Alg.

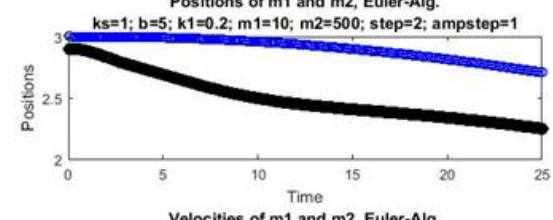

Velocities of $\mathrm{m} 1$ and $\mathrm{m} 2$, Euler-Alg.

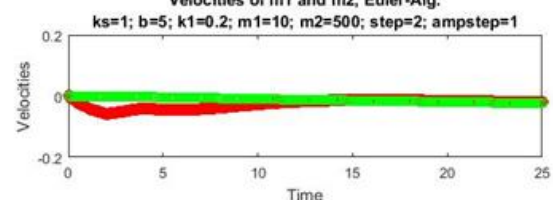

Positions of $\mathrm{m} 1$ and $\mathrm{m} 2$, Euler-Alg.
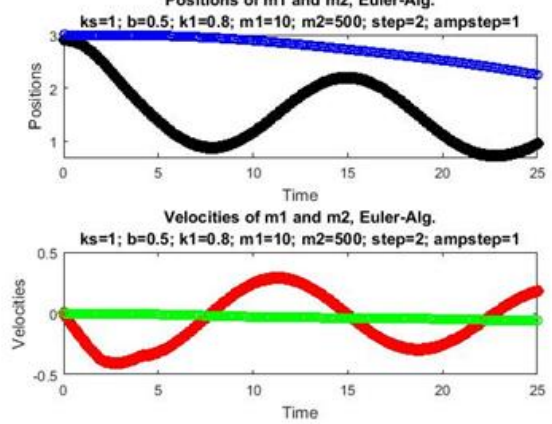

Fig. 7: Positions and velocities of $m_{1}$ and $m_{2}$ when $K_{s}$, b, and $K_{1}$ are varied respectively, when the vehicle hits a bump in the road whose profile is shown in the next figure.

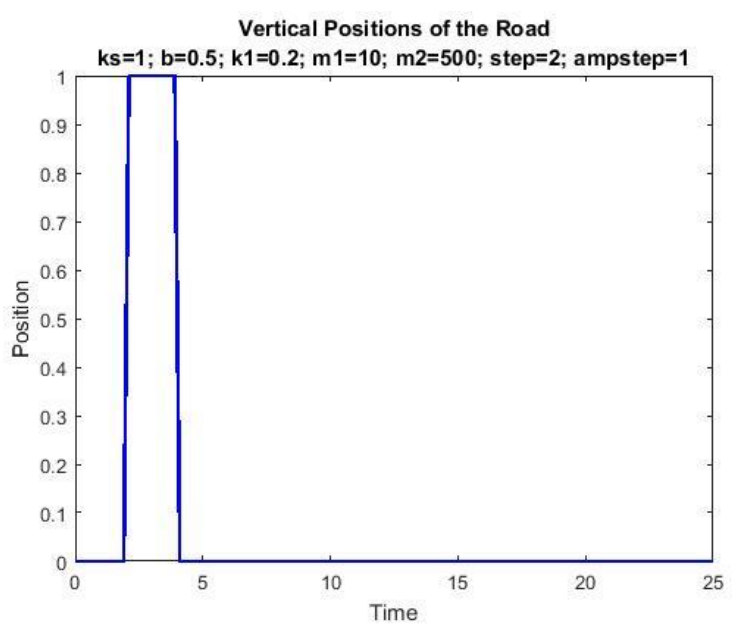

Fig. 8: Profile of a bump in the road used in the numerical solution of the car suspension model whose results are shown in the previous figure. 


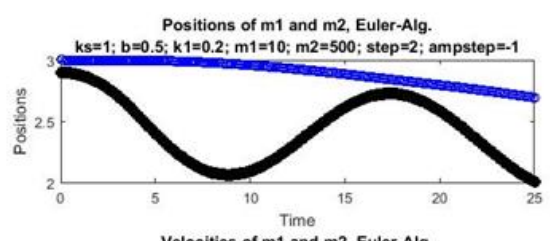

Velocities of $\mathrm{m} 1$ and $\mathrm{m} 2$, Euler-Alg.

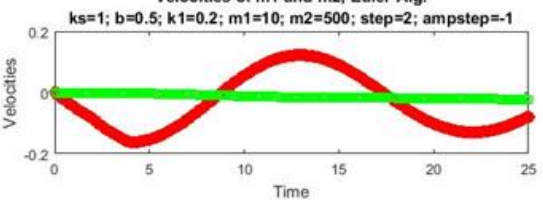

Positions of $\mathrm{m} 1$ and $\mathrm{m} 2$, Euler-Alg.

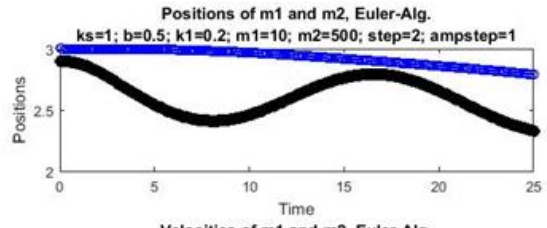

Velocities of $\mathrm{m} 1$ and $\mathrm{m} 2$, Euler-Alg.

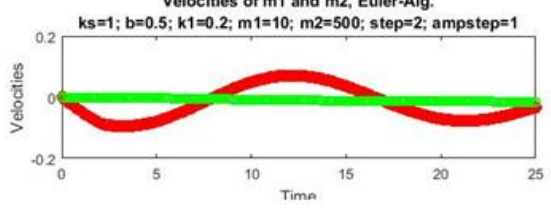

Positions of $\mathrm{m} 1$ and $\mathrm{m} 2$, Euler-Alg.
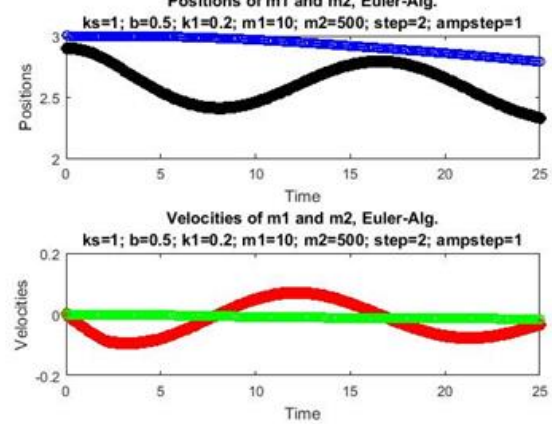



Velocities of $\mathrm{m} 1$ and $\mathrm{m} 2$, Euler-Alg.

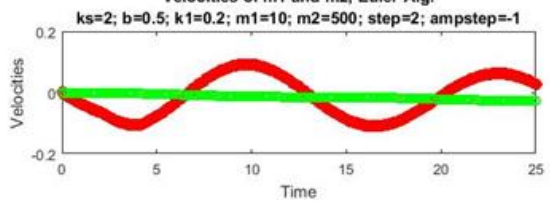

Positions of $\mathrm{m} 1$ and $\mathrm{m} 2$, Euler-Alg.
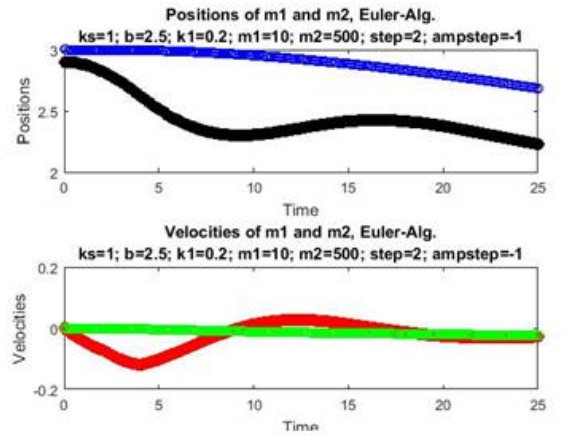

Positions of $\mathrm{m} 1$ and $\mathrm{m} 2$, Euler-Alg.

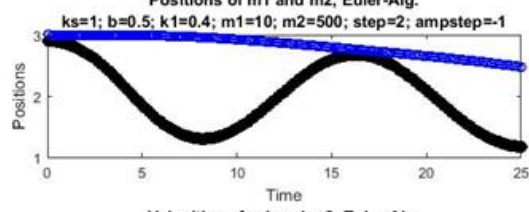

Velocities of $\mathrm{m} 1$ and $\mathrm{m} 2$, Euler-Alg.

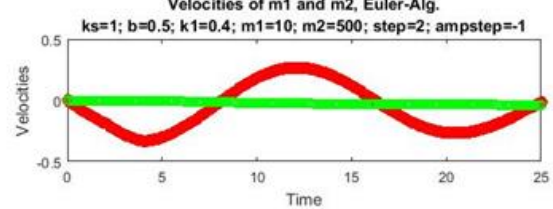

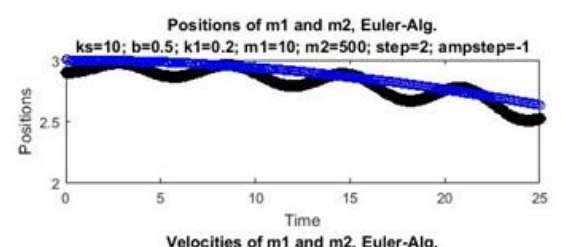

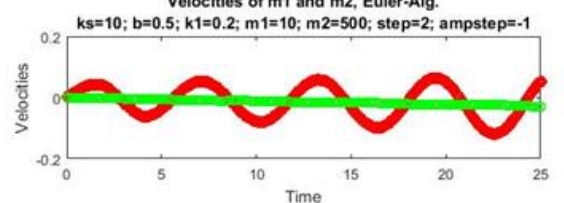

Positions of $m 1$ and $m 2$, Euler-Alg
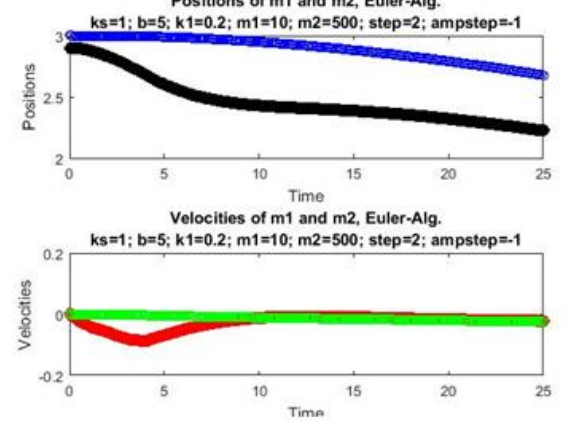

Positions of $m 1$ and $m 2$, Euler-Alg.
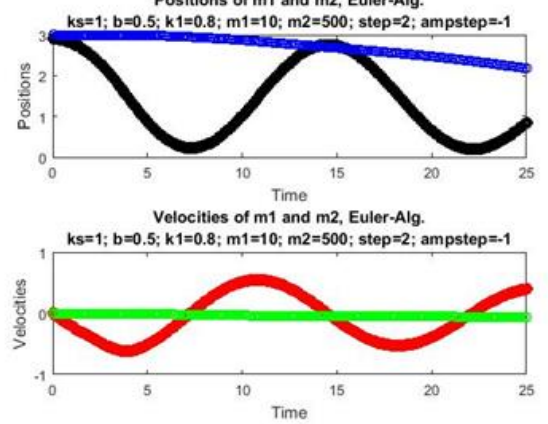

Fig. 9: Positions and velocities of $m_{1}$ and $m_{2}$ when $K_{s}$, b, and $K_{1}$ are varied respectively, when the vehicle hits a hole in the road whose profile is shown in the next figure.

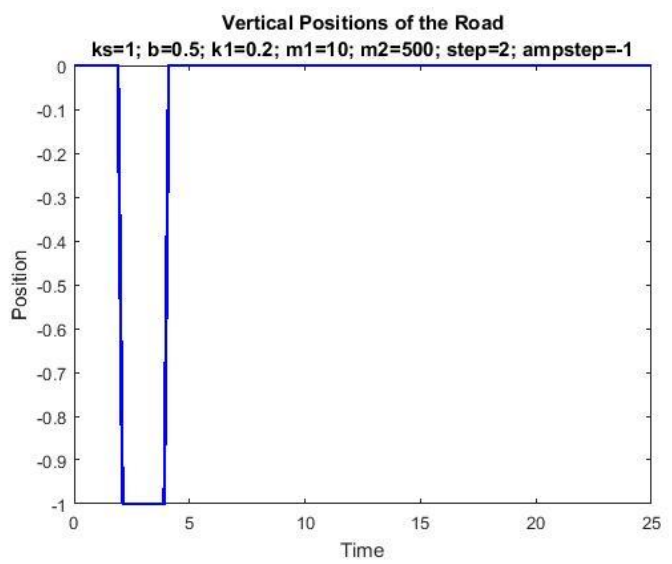

Fig. 10: Profile of a hole in the road used in the numerical solution of the car suspension model whose results are shown in the previous figure. 
Vertical Positions of the Road

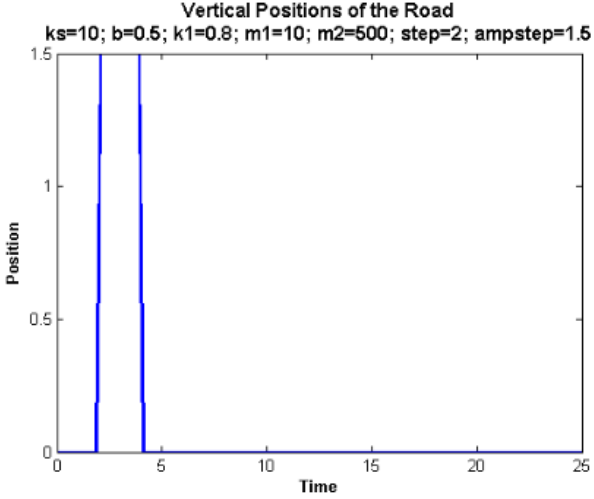

Positions of $\mathrm{m} 1$ and $\mathrm{m} 2$, Euler/RK2/RK4 Algorithms $k s=10 ; b=0.5 ; k 1=0.8 ; m 1=10 ; m 2=500 ;$ step=2; ampstep $=1.5$

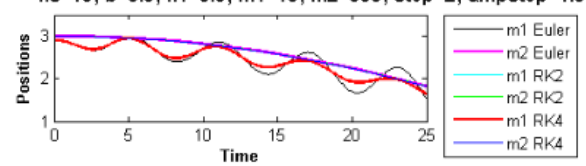

Velocities of $m 1$ and $m 2$, Euler/RK2/RK4 Algorithms $\mathrm{ks}=10 ; \mathrm{b}=0.5 ; \mathrm{k} 1=0.8 ; \mathrm{m} 1=10 ; \mathrm{m} 2=500 ; \mathrm{step}=2 ;$ ampstep $=1.5$

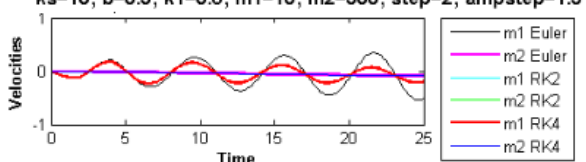

Vertical Positions of the Road



Positions of $m 1$ and $m 2$, Euler/RK2/RK4 Algorithms $\mathrm{ks}=10 ; \mathrm{b}=0.5 ; \mathrm{k} 1=0.8 ; \mathrm{m} 1=10 ; \mathrm{m} 2=500 ; \mathrm{step}=2 ; \mathrm{ampstep}=1.5$

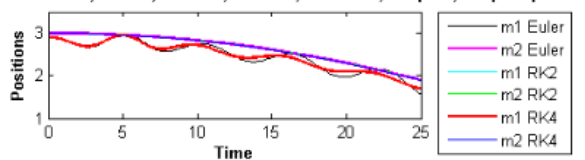

Velocities of $m 1$ and $m 2$, Euler/RK2/RK4 Algorithms $\mathrm{ks}=10 ; b=0.5 ; \mathrm{k} 1=0.8 ; \mathrm{m} 1=10 ; \mathrm{m} 2=500 ; \mathrm{step}=2 ;$ ampste $=1.5$

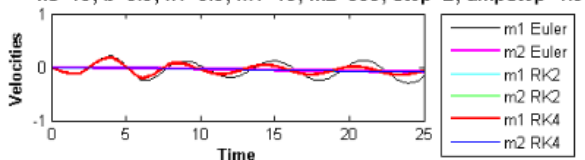

Vertical Positions of the Road

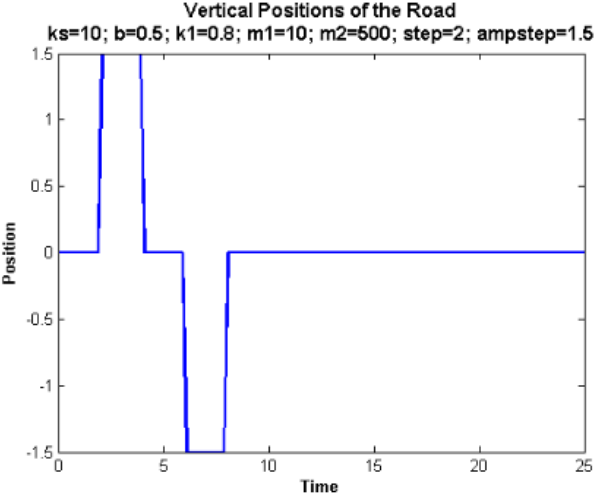

Positions of $\mathrm{m} 1$ and $\mathrm{m} 2$, Euler/RK2/RK4 Algorithms $k s=10 ; b=0.5 ; k 1=0.8 ; m 1=10 ; m 2=500 ;$ step $=2 ;$ ampstep $=1.5$

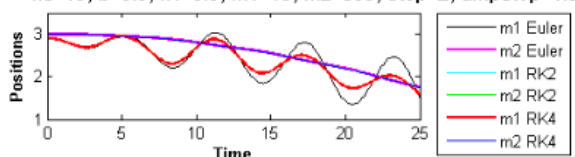

Velocities of $\mathrm{m} 1$ and $\mathrm{m} 2$, Euler/RK2/RK4 Algorithms $k s=10 ; b=0.5 ; k 1=0.8 ; m 1=10 ; m 2=500 ;$ step=2; ampstep=1.5

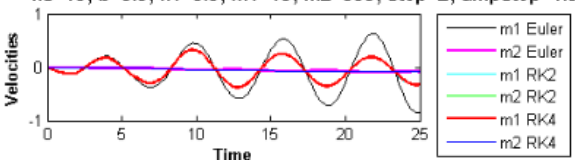

Fig. 11: Positions and velocities of when the vehicle hits a bump in the road, or two bumps or a bump then a hole, with a separation of 2 and step amplitude of 1.5 .
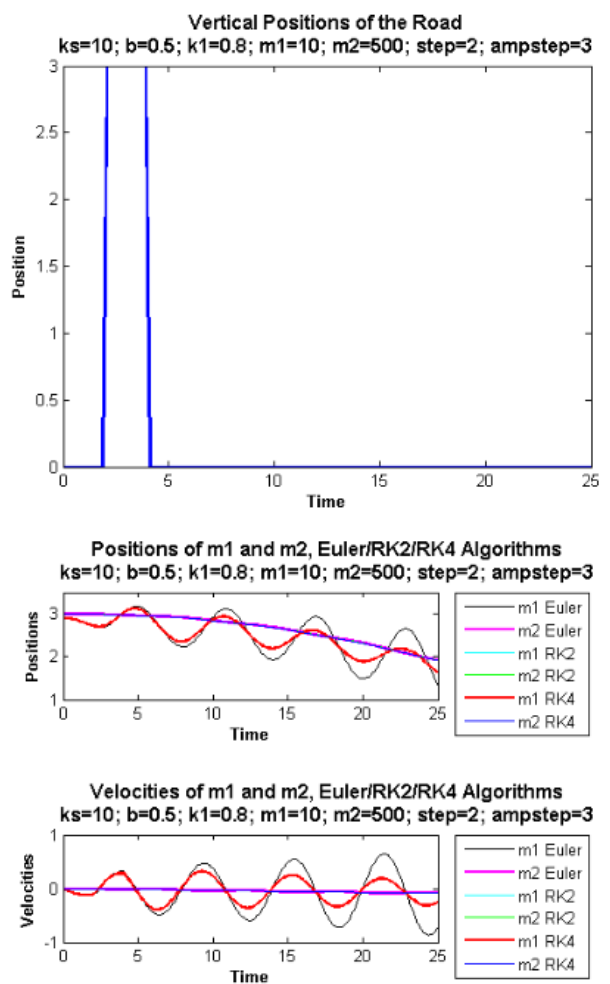
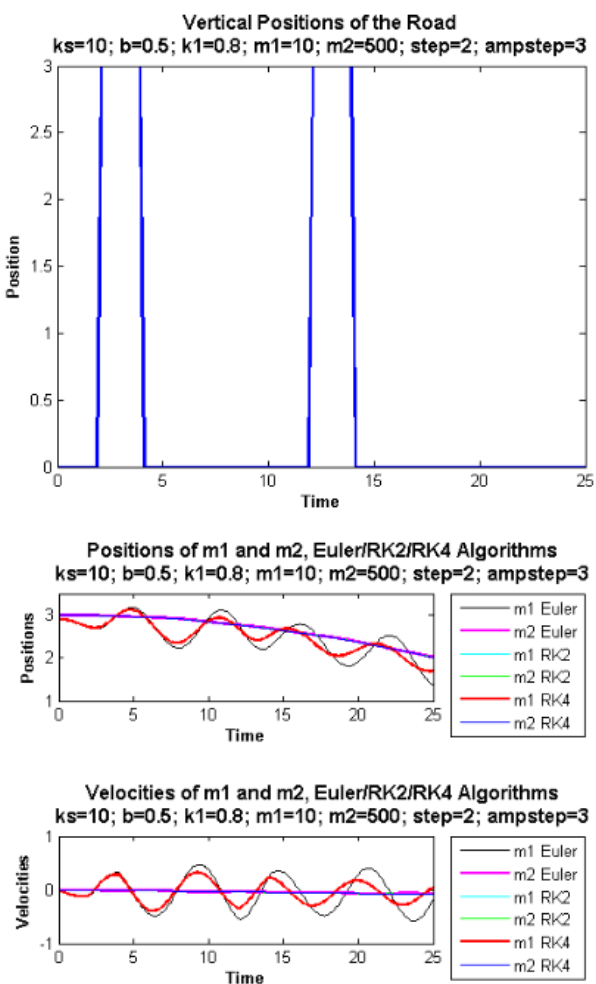

Vertical Positions of the Road

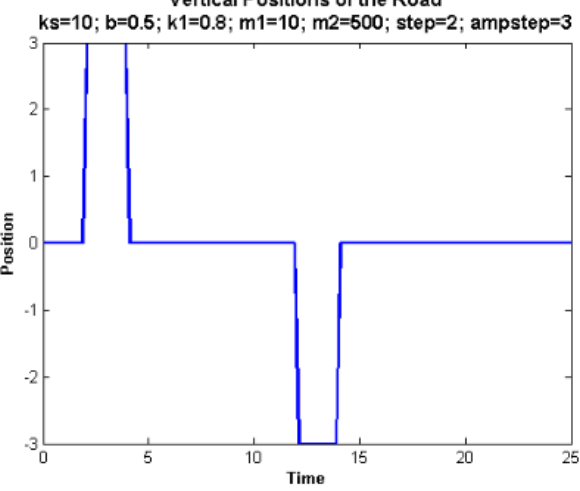

Positions of $\mathrm{m} 1$ and $\mathrm{m} 2$, EuleriRK2/RK4 Algorithms $\mathrm{ks}=10 ; \mathrm{b}=0.5 ; \mathrm{k} 1=0.8 ; \mathrm{m} 1=10 ; \mathrm{m} 2=500 ;$ step $=2 ;$ ampstep $=3$

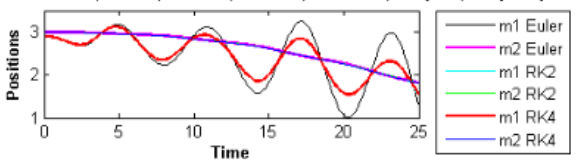

Velocities of $\mathrm{m} 1$ and $\mathrm{m} 2$, Euler/RK2/RK4 Algorithms

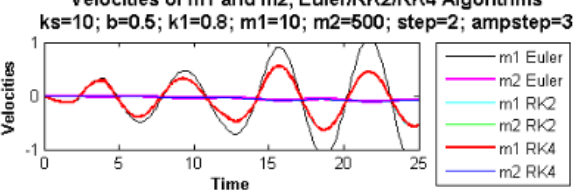

Fig. 12: Positions and velocities, when the vehicle hits a bump in the road, or two bumps or a bump then a hole, with a separation of 10 and step amplitude of 3. 


\section{Animations}

Since our method solves the suspension model in the time domain, animations are made of the coupled motions of the car and its axle/suspension as it hits different types of obstacles in the road. An example was shown in Figure 4.

\section{Conclusion}

Car suspension was studied, using the Quarter Car Suspension Model, which consists of a suspension spring and damper (coil and shock absorber) between the sprung mass (body of the car where passengers are seated) and the un-sprung mass (half-axle, suspension, and wheel), in series with tire spring between un-sprung mass and the road. Newton's Second Law for the sprung and un-sprung masses lead to a system of two coupled second-order differential equations (ODEs). The system is rewritten as a system of four coupled first-order differential equations. The system was solved numerically in the time domain using the Euler's leapfrog method for first-order ODEs. After studying the results using road conditions such as steps, bumps, and holes the following observations were made: using a smaller suspension spring and tire spring constant, a larger damping constant, those values leading to less oscillations of the un-sprung mass. RK2 and RK4 were implemented and compared with Euler's method for different bump height, bump-bump, and bump-hole interactions. The advantage of the higher order methods were clearly seen at larger time steps and around the local extremes of the velocity curves. The results agreed with the intuition that it was more difficult to go over taller bumps or deeper holes, or having bump-bump or bump-hole separation that is closest to the length between the front and back car suspension. Future work will include the study of the effect on the vehicle's speed limit to avoid the bottom-out scenario, and the study of four interacting Quarter Car Suspensions for a given vehicle.

\section{Acknowledgements}

Special thanks to Edward Pang for his time and feedback on my discovery process and the writing of the paper. Thanks to Ahaan Rungta for directing the Junction program and thanks to the ESP-MIT staff for the support. Thanks to MIT for letting me use the facilities.

\section{References}

[1] D. G. Alciatore, Introduction to Mechatronics and Measurement Systems, 3rd ed., McGraw Hill, 2007.

[2] W. Press, S. Teukolsky, W. Vetterling, and B. Flannery, Numerical Recipes $3^{\text {rd }}$ Ed.: The Art of Scientific Computing. Cambridge University Press, 2007.

[3] S. Kashem, and R. Nagarajah, Vehicle Suspension Systems and Electromagnetic Dampers. Springer, 2017.

[4] M. Tenenbaum, and H. Pollard, Ordinary Differential Equations. Harper \& Row, 1963.

[5] S. J. Farlow, Partial Differential Equations for Scientists and Engineers. Dover, 2012.

[6] R. O. Da Souza, et al, Improving Pavements with Long-Term Pavement Performance: Products for Today and Tomorrow Study of Long-Term Pavement Performance (LTPP): Pavement Deflections Paper 1: Statistical analysis between roughness indices and roughness Prediction model using neural networks. [Online]. Available: www.fhwa.dot.gov/publications/research/ infrastructure/pavements/ltpp/06109/paper1.cfm

[7] S. H. Strogatz, Nonlinear Dynamics and Chaos, 2nd ed. CRC Press, 2018.

[8] Typical Front Wheel Drive. [Online]. Available: i.pinimg.com/originals/36/b7/a0/36b7a07ab676b00351142a3b9d8c50fe.jpg 